Review Article

\title{
Nanocomposites of Graphene with Ferrocene or Hemin: Preparation and Application in Electrochemical Sensing
}

\author{
Ning Xia, Lin Liu, Zhifang Sun, and Binbin Zhou \\ College of Chemistry and Chemical Engineering, Anyang Normal University, Anyang, Henan 455000, China \\ Correspondence should be addressed to Lin Liu; liulin@aynu.edu.cn and Binbin Zhou; bbzhou1985@163.com
}

Received 20 January 2015; Revised 3 March 2015; Accepted 5 March 2015

Academic Editor: Jian Gong

Copyright ( 2015 Ning Xia et al. This is an open access article distributed under the Creative Commons Attribution License, which permits unrestricted use, distribution, and reproduction in any medium, provided the original work is properly cited.

\begin{abstract}
Graphene has sparked tremendous interests across many fields such as biosensors, electrochemical energy storage, and electronics due to its extraordinary physicochemical and structural properties. The redox mediator-functionalized graphene composites may obtain highly electroactive materials with better conductivity, dispersion, and compatibility. Ferrocene and hemin, two iron-organic complexes, are the well-known mediators in view of their various properties, including reversibility, regeneration at low potential, and generation of stable redox states. Recently, it has been demonstrated that the use of ferrocene or hemin coupled with graphene can further improve the electrochemical performances of the developed sensors. In this contribution, we, for the first time, reviewed the preparation and applications of ferrocene/graphene and hemin/graphene nanocomposites in electrochemical sensing.
\end{abstract}

\section{Introduction}

Graphene is a single-atom-thick planar sheet of sp2-bonded carbon atoms with exciting properties such as extremely high electronic conductivity and superior mechanical strength $[1,2]$. Because of its extraordinary physicochemical and structural properties, this exciting new material has quickly sparked tremendous interests across many fields, including biosensors, electrochemical energy storage, and electronics. With these added advantages, it is desirable to harness the useful properties of graphene and its derivatives in composites through the incorporation with various kinds of functional materials such as inorganic nanostructures, organic crystals, polymers, metal-organic frameworks (MOFs), biomaterials, and carbon nanotubes (CNTs). Properties, preparations, and applications of these functionalized graphene hybrid materials have been summarized in many review papers [3-11]. It is well known that electron mediator is highly conductive molecule, having a readily accessible redox couple and working as the electron transfer mediator to shorten the electron transfer distance between the redox center of analyte and electrode surface. Accordingly, redox mediator may be useful for construction of novel graphene-based nanomaterials. The redox mediator-functionalized graphene composites may obtain highly electroactive materials with better conductivity, dispersion, and compatibility, which made them highly desirable for effective application in electrochemical sensing. Ferrocene and hemin, two iron-organic complexes, are the well-known mediators in view of their various properties, such as reversibility, regeneration at low potential, and generation of stable redox states. Recently, it has been demonstrated that the use of ferrocene or hemin coupled with graphene can further improve the electrochemical performances of the developed sensors. To the best of our knowledge, there is no review paper focusing on the preparation and application of ferrocene/graphene and hemin/graphene nanocomposites. In this paper, we addressed the preparation and emerging applications of graphene loaded with ferrocene or hemin for electrochemical sensing.

\section{Ferrocene/Graphene Nanocomposites}

2.1. Preparation and Applications of Ferrocene/Graphene Modified Electrode. The commonly used methods for modification of ferrocene or its derivatives on graphene include $\pi-\pi$ stacking interaction, covalent bonding, and physical entrapment. The method based on $\pi-\pi$ stacking interaction is an 


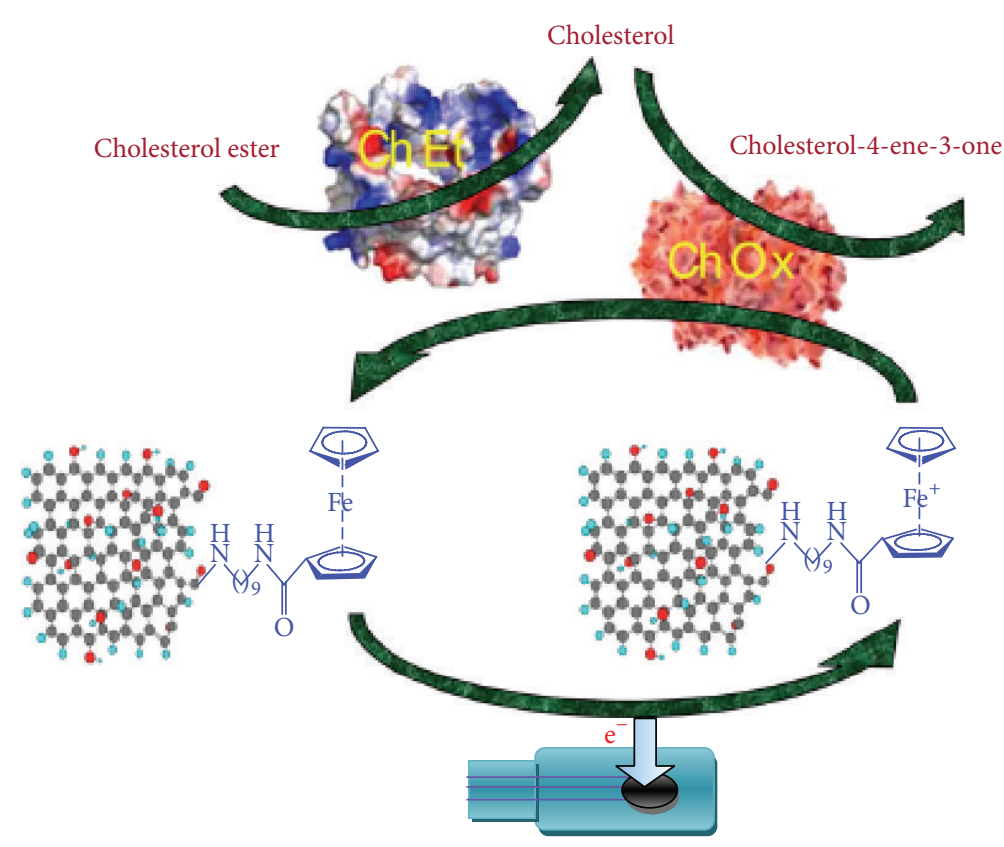

(a)

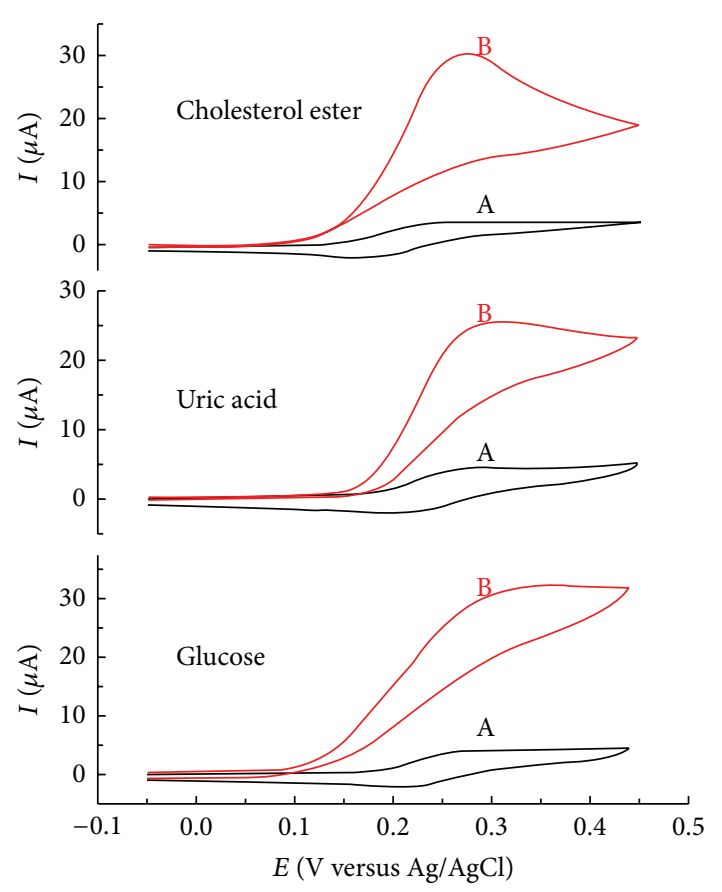

(b)

FIGURE 1: (a) Scheme illustrating the biosensing of cholesterol ester mediated by Fc-GO on SPE. (b) Cyclic voltammetric response of the biosensors toward cholesterol ester, uric acid, and glucose in the (A) absence and (B) presence of the respective analytes. (Reprinted with permission from [16]. Copyright 2013 American Chemical Society).

easy means for preparation of ferrocene/graphene nanocomposites [12-14]. By such interaction, the ferrocene/graphene modified graphite electrode has been prepared for the simultaneous electrochemical determination of captopril and hydrochlorothiazide [13]. Furthermore, Deng et al. investigated the electron transfer behaviors of ferrocene/reduced graphene oxide (rGO) on glass carbon electrode and demonstrated its application in detection of $\mathrm{H}_{2} \mathrm{O}_{2}$ and choline using chitosan for the immobilization of horseradish peroxidase (HRP) and choline oxidase (CHOD) [14]. However, the $\pi-\pi$ stacking interaction between the graphene and ferrocene may result in low sensitivity and stability of the biosensor because ferrocene, especially in the oxidized form, can easily diffuse away from the electrode surface into the bulk solution. Therefore, the leakage of electron shuttling ferrocene is a main problem for the modified electrodes. Alternatively, covalently bonded method is a more effective modification means for preparation of ferrocene/graphene nanocomposites [15]. The reported methods for covalent attachment of ferrocene derivatives to graphene include carbodiimidemediated amine coupling, Schiff's base reaction, and FriedelCrafts monoacylation. Among them, carbodiimide provides the most popular method for the immobilization of ferrocene derivatives. For example, Dey and Raj demonstrated that ferrocenecarboxylic acid ( $\mathrm{FcA}$ ) could be covalently attached onto the graphene oxide (GO) backbone with diamine sigma spacers of different chain lengths (C3-, C6-, and C9diamines) and the surface coverage as well as heterogeneous electron transfer rate constant of FcA-GO depended on the length of sigma spacer (Figure 1) [16]. The screen printed electrodes modified with FcA-GO and the respective redox enzymes allowed for the sensitive detection of cholesterol, uric acid, and glucose. In the process, FcA-GO efficiently mediated the bioelectrocatalytic oxidation of the substrates in the presence of the redox enzymes. Additionally, Liu et al. reported the synthesis of an electroactive graphene composite by covalent immobilization of an amine-terminated ferrocene derivative (4-ferrocenylethyne phenylamine, FEPA) on GO through the interaction between the $-\mathrm{NH}_{2}$ groups of FEPA and the $-\mathrm{COOH}$ groups of GO. Compared with the alkyl ferrocene derivatives, the "chain-like" FEPA molecule possesses lower resistance and higher electric conductivity. The resulting chitosan/FEPA-GO/GCE showed high sensitivity and selectivity for nitrite detection [17]. Schiff's base reaction is another means for the covalent coupling of ferrocene derivatives to graphene. Typically, ferrocenecarboxaldehyde has been immobilized onto the ethylenediamine- (ED-) functionalized GO through Schiff's base reaction $[18,19]$. The resulting ferrocene/graphene nanocomposites showed high electrochemical catalytic ability to the oxidation of $\mathrm{H}_{2} \mathrm{O}_{2}$ [19] and effective electrochemiluminescence quenching pattern to tris $\left(2.2^{\prime}\right.$-bipyridyl)ruthenium (II) $\left[\mathrm{Ru}(\mathrm{bpy})_{3}{ }^{2+}\right][18]$. Moreover, Avinash et al. reported the covalent functionalization of GO with ferrocene via the selective Friedel-Crafts monoacylation of ferrocene [20]. This method provided an alternative means for preparation of ferrocene/graphene nanocomposites.

Beside the $\pi-\pi$ stacking interaction and covalent bonding, entrapment in ionic liquid (IL), polymers (e.g., nafion, chitosan), or sol-gel matrices provides a unique means 
to prepare a three-dimensional network suited for the encapsulation of ferrocene and/or enzymes. IL is a compound consisting entirely of ions that exist in liquid state around room temperature. Recently, IL has been investigated as a biocompatible material for the fabrication of biosensors because of its high chemical and thermal stability, good conductivity, wide electrochemical windows, and good dissolving capability [21]. It has been demonstrated that the hemoglobin/chitosan-IL-ferrocene/graphene modified glassy carbon electrode showed a strong electrocatalytic activity toward the reduction of $\mathrm{H}_{2} \mathrm{O}_{2}$ due to the favored orientation of hemoglobin in the well-confined surface as well as the high electrical conductivity of the resulting chitosan-IL-ferrocene/graphene inorganic hybrid composite [22]. Moreover, Tajik et al. reported the simultaneous electrocatalytic determination of isoproterenol, acetaminophen, and theophylline using a 1-(4-bromobenzyl)-4-ferrocenyl-1H$[1,2,3]$-triazole- (1,4-BBFT-) IL/graphene modified electrode [23].

The grafting of polymers onto graphene is also one of effective methods for the preparation of polymer composites with graphene. Therefore, modification by grafting of polymers onto graphene has been reported by many researchers $[24,25]$. For example, nafion's superior properties make it suitable for a broad range of applications; Liu et al. suggested that the presence of nafion and 4-ferrocenylethyne phenylamine (FEPA) in the graphene composite not only provided an excellent environment for dopamine oxidation but also acted as a barrier to prevent ascorbic acid from interfering in the dopamine detection [26]. For low molecular weight mediator compounds, leakage is the main problem in their entrapment in sol-gel matrices. Usually, this limitation can be resolved through direct cross-linking of ferrocene derivatives with polymer, nanoparticles, or high molecular weight compounds before immobilization. For this view, Peng et al. reported the preparation of ferrocene-conjugated ormosil by Schiff's base reaction between the aldehyde group of ferrocenecarboxaldehyde and the amino group of the sol-gel precursor 3-(aminopropyl)triethoxysilane (APTES) [27]. Tetraethoxysilane (TEOS) and the synthesized APTESferrocene were then used as the precursors for the solgel polymerization. The functional TEOS-APTES-ferrocene composite could effectively prevent the leakage of Fc and showed high stability. As a result, a glucose biosensor was developed by entrapping glucose oxidase (GOD) in the Fcbranched ormosil/chitosan/GO nanocomposites.

Very recently, Fatoni et al. presented a novel molecularly imprinted polymer cryogel biosensor for determination of microalbumin in urine samples by entrapping ferrocene and graphene in the cryogel [28]. Specially, the molecularly imprinted polymer gel was prepared based on the graft copolymerization of acrylamide with $\mathrm{N}, \mathrm{N}^{\prime}$ methylenebisacrylamide on chitosan using human serum albumin as the template. The subzero polymerization allowed the solvent to form ice crystals and left a macroporous cryogel structure when it was thawed. After removing the template, the specific imprinted surface on cryogel pore walls was used to detect human serum albumin.
2.2. Ferrocene/Graphene/Nanoparticles Hybrid Materials for Electrochemical Sensing. The integration of nanomaterials on graphene potentially paves a new way to enhance their electronic, chemical, and electrochemical properties. Recently, several groups have reported the preparation and application of ferrocene/graphene/nanoparticles hybrid materials. Typically, Han et al. developed a label-free amperometric immunosensor for detection of carcinoembryonic antigen based on chitosan-ferrocene/nano- $\mathrm{TiO}_{2}$ complex film and AuNPs-graphene nanohybrid. In the work, the AuNPs-graphene nanohybrid was formed on the chitosanferrocene/nano- $\mathrm{TiO}_{2}$-modified electrode by self-assembly [29]. Liu et al. reported the detection of rutin on ferrocene/AuNPs/graphene sheet-chitosan modified glass carbon electrode [30]. Lately, they presented the fabrication of the nanocomposites of ferrocene thiolate stabilized $\mathrm{Fe}_{3} \mathrm{O}_{4} @ \mathrm{Au}$ nanoparticles with graphene sheet/chitosan on a glassy carbon electrode (GCE) surface [31]. The modified electrode allowed for the ultrasensitive and simultaneous detection of ascorbic acid, dopamine, uric acid, and acetaminophen. Using a homogeneous three-component electrodeposition solution containing a ferrocene grafted chitosan hybrid, GOD, and single-walled carbon nanotubes (SWNTs), a homogeneous biocomposite film of ferrocenechitosan/SWNTs/GOD was immobilized on the surface of 3D graphene foam by one-step electrodeposition [32]. The ferrocene groups grafted on chitosan were stably immobilized on the 3D graphene surface and keep their original electrochemical activity. The SWNTs doped into the ferrocenechitosan matrix acted as a nanowire to facilitate electron transfer and improved the conductivity of the biocomposite film. Furthermore, a signal amplification detection platform based on the electrochemical rGO-ferrocene hybrid nanocomposite and AuNPs was established for detection of bisphenol A. In the work, the amine-terminated ferrocene derivative was hybridized with $\mathrm{GO}$ through covalent coupling reaction. After coupling with $\mathrm{HAuCl}_{4}$ to simultaneously electrodeposit on the glassy carbon electrodes, the rGOferrocene/AuNPs modified electrode was obtained [33].

More interestingly, Wan et al. reported the fabrication of a novel magnetically driven fuel-free graphene carrier loaded with ferrocene (Fc) (Figure 2) [34]. The activation and deactivation of redox-active cargo Fc's redox activity by magnetically driven positioning of the graphene carrier with loaded Fc near and away from the conductive support can be employed for magneto-switchable bioelectrocatalyzed oxidation of glucose by glucose oxidase between active and inactive electrocatalytic states, respectively. The artificially magneto-triggered bioelectrocatalysis and modulation of the electron transfer process of redox biomolecules are extremely important for understanding electron-transport pathways in magneto-triggered biosystems and for mimicking the dynamic properties of magneto-sensitive biochemical reactions in real bioprocesses.

\section{Hemin/Graphene Nanocomposites}

Hemin is a well-known protoporphyrin found at the active site of heme protein and plays a key role in biochemical 


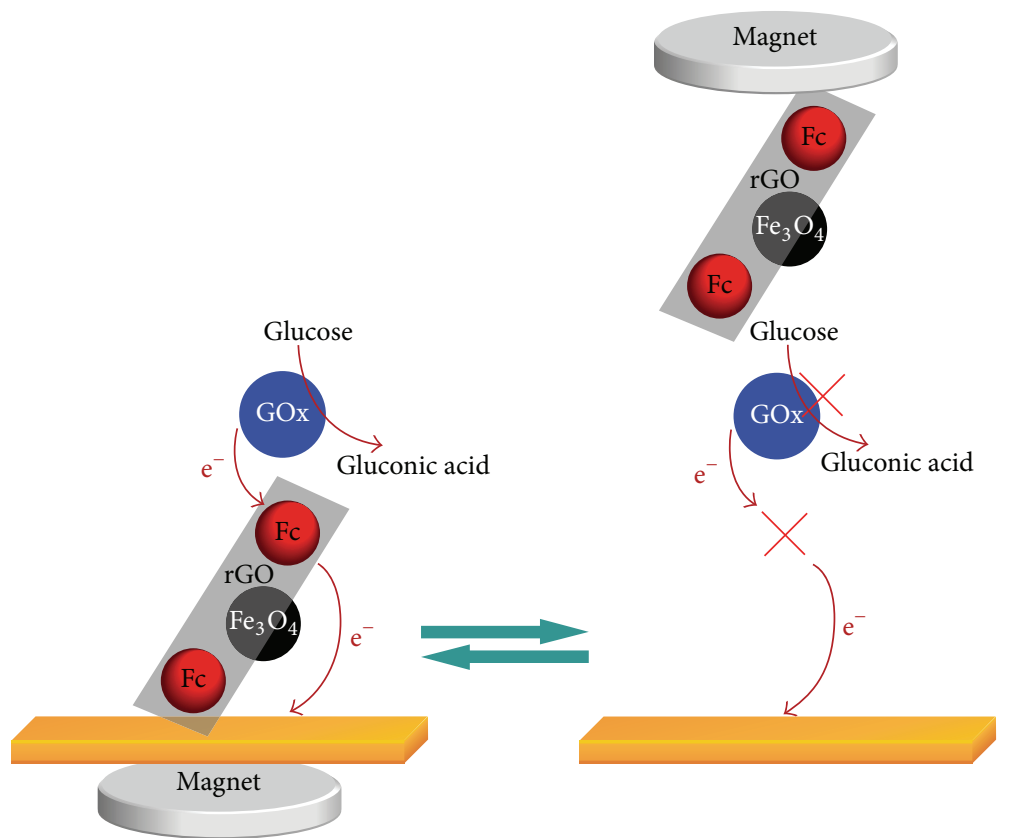

(i)

(ii)

(a)

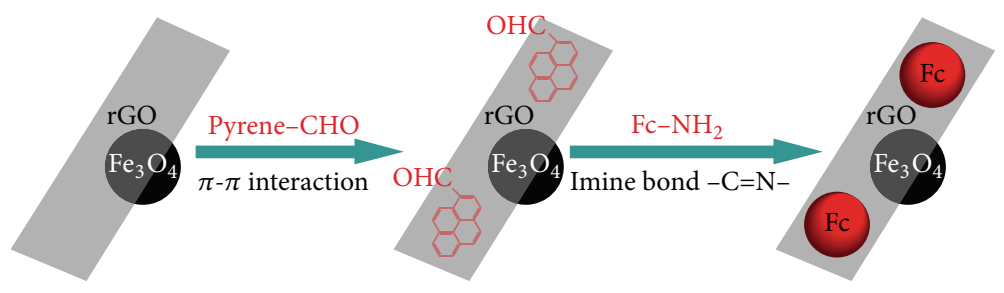

(b)

FIGURE 2: (a) Magnetically driven fuel-free graphene carrier with loading of redox-active cargo-Fc working as an electron mediator for mediated bioelectrocatalysis of glucose to gluconic acid by GOx switched between "on" and "off" states under alternate positioning of the graphene carrier with loaded Fc (i) near and (ii) away from the conductive support. (b) The loading of Fc onto the graphene carrier by $\pi-\pi$ interaction between $\mathrm{Py}-\mathrm{CHO}$ and the unoccupied areas of the graphene nanosheet, followed by the formation of imine bond $(-\mathrm{C}=\mathrm{N}-$ ) between $-\mathrm{CHO}$ group of the $\mathrm{Py}-\mathrm{CHO}$ modified graphene carrier and the $-\mathrm{NH}_{2}$ group of the $\mathrm{Fc}-\mathrm{NH}_{2}$. (Reprinted with permission from [34]. Copyright 2014 John Wiley and Sons).

reaction and electron-transport chain $[35,36]$. Hemin can be well used as electron media based on the reversible redox of $\mathrm{Fe}(\mathrm{III}) / \mathrm{Fe}(\mathrm{II})$ and hemin-functionalized graphene nanosheets have been shown to exhibit peroxidase activity recently. The preparation and applications of hemin/ graphene nanocomposites in electrochemical sensing were also summarized herein.

3.1. Preparation and Applications of Hemin/Graphene Modified Electrode. Among the efforts, the technique of immobilizing hemin on graphene through aromatic $\pi-\pi$ interaction was found to be very promising due to its enhanced electron transfer, enhanced electrostatic interaction, excellent electrocatalytic response, peroxidase-like activity, and easy preparation. Typically, conducting interfaces modified with rGO have shown improved electrochemical response for different analytes. The hemin-functionalized rGO hybrid nanomaterials have been prepared for development of sensitive electrochemical sensors in several groups. For example, Guo et al. reported the first dual biosensor platforms for detection of $\mathrm{H}_{2} \mathrm{O}_{2}$ and glucose based on $\mathrm{rGO}$ /hemin nanosheets [37]. The synergic effect between graphene (high conductivity and surface area) and hemin (excellent catalysis and intrinsic peroxidase-like activity) led to highly efficient electrocatalytic activity for the reduction of $\mathrm{H}_{2} \mathrm{O}_{2}$ at the rGO/hemin nanosheets modified glassy carbon electrode and exhibited intrinsic peroxidase-like activity that catalyzed $\mathrm{H}_{2} \mathrm{O}_{2}$ to oxidize 3,3,5,5-tetramethylbenzidine (TMB) to produce a blue color reaction. Also, Zhang et al. presented the electrogenerated chemiluminescence biosensors for the determination of cholesterol by modifying cholesterol oxidase (ChOx) on the $\mathrm{rGO} /$ hemin nanosheets [38, 39]. In above works, rGO was prepared by the chemical reduction of GO with hydrazine. Chemical reduction of GO with $\mathrm{NaBH}_{4}$, 


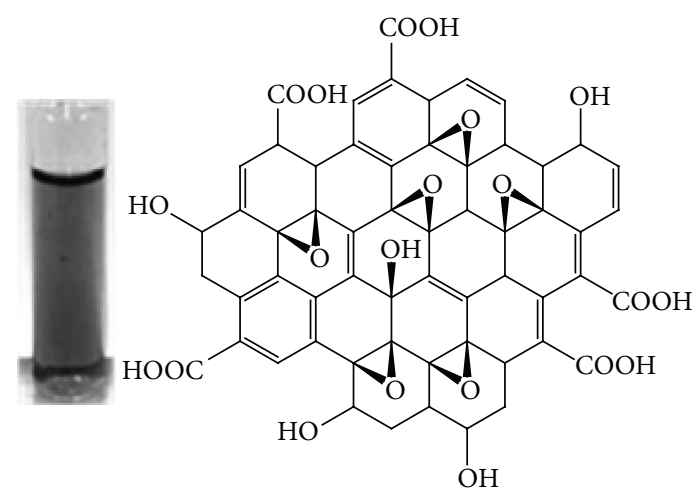

GO
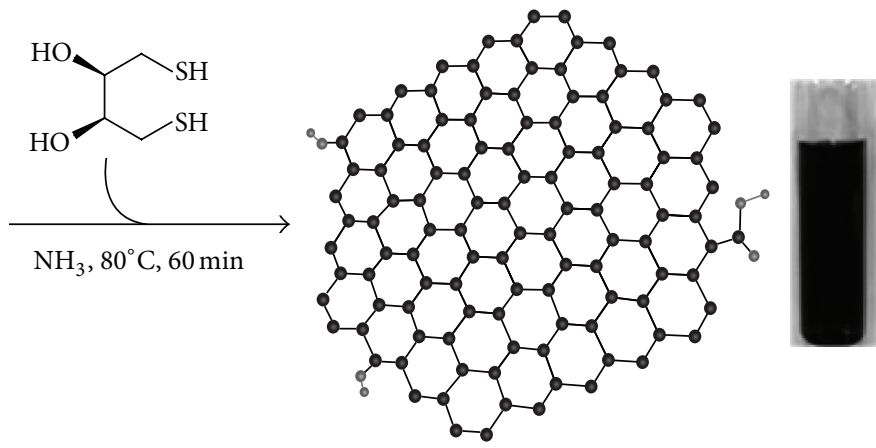

rGO

Figure 3: Schematic representation of the synthesis of rGO by the reduction using DTT. (Reprinted with permission from [40]. Copyright 2012 John Wiley and Sons).

hydrazine, hydroquinone, dimethyl hydrazine, and $\mathrm{Fe} / \mathrm{HCl}$ has marked an impact on the generation of copious quantity of rGO with cost effectiveness. However, great care is essential while using these highly toxic and explosive reducing agents [40]. Moreover, functionalization of graphene with polar molecules imparts the hydrophilicity over hydrophobic effects, thus enhancing its dispersibility in polar solvents. For these views, new reducing reagents or reduction methods have been developed for the preparation of graphenebased nanocomposites [41, 42]. Typically, dithiothreitol is a remarkably strong reducing agent with redox potential of $-0.33 \mathrm{~V}$ at $\mathrm{pH} 7$; Vernekar et al. reported the reduction of GO using dithiothreitol as the reducing agent. The noncovalent functionalization of rGO with hemin resulted in hemin/rGO hybrid material that exhibited a remarkable antioxidant activity for reduction of peroxynitrite (Figure 3) [40]. It has also been demonstrated that $\mathrm{GO}$ can be easily reduced using aromatic organic molecules under mild conditions. Oprea et al. found that hemin allowed for the simultaneous reduction of and incorporation into GO nanosheets and that the resulting $\mathrm{rGO} /$ hemin showed high peroxynitrite activity [43]. Tang et al. reported a photopolymerization method to prepare $\mathrm{rGO} /$ bovine serum albumin (BSA) cross-linked polymer hydrogel/hemin nanocomposites [44]. The GO nanosheets were adsorbed and reduced in situ during the irradiation. The formation of rGO during the UV irradiation and polymerization is mainly contributed to the tyrosine residues of BSA. The large-scale synthesis of ternary nanocomposites of hemin-rGO/poly(3,4-ethylenedioxythiophene) was achieved via a microwave-assisted method [45], in which poly(3,4ethylenedioxythiophene) was induced with hemin and polymerized with GO and GO was partially reduced. The method did not require the additional oxidant for polymerization and reducing reagents for reduction of GO. The synthesized nanocomposites also exhibited good biocompatibility, fast redox property, and remarkable electrocatalysis towards the reduction of $\mathrm{H}_{2} \mathrm{O}_{2}$. Moreover, a hydrothermal method was reported by Wei et al. as a safe and green chemistry route for the reduction of GO. The prepared $\mathrm{rGO} /$ hemin nanosheets were used for the electrochemical detection of L-tyrosine [42].
3.2. Hemin/Graphene/Nanoparticles Hybrid Materials for Electrochemical Sensing. Recently, hemin/graphene/nanoparticles hybrid materials have also been used in several sensing and catalytic applications with encouraging results $[46,47]$. For example, AuNPs have a large surface area and good conductivity and enable the loading of a large amount of hemin-rGO and the promotion of electron transfer; the layerby-layer hemin-rGO/AuNPs structure significantly improved the electrocatalytic activity and the electrochemical stability for the determination of $\mathrm{H}_{2} \mathrm{O}_{2}$ with a detection limit of $0.11 \mu \mathrm{M}$ (Figure 4) [46]. At the same time, Zhang et al. reported a simple wet-chemical strategy for synthesizing hemin/GO/carbon nanotubes nanocomposites through the $\pi-\pi$ interactions [47]. The hybrid materials were used to construct a novel dual sensor for the determination of $\mathrm{H}_{2} \mathrm{O}_{2}$ and the simultaneous determination of ascorbic acid, dopamine, uric acid, and tryptophan. The success of these initial efforts provides good motivation for further manipulation of hemin/graphene/nanoparticles hybrid materials to optimize sensing and catalytic efficiencies.

3.3. Hemin-Loaded Graphene as the Signal Label. It is well known that hemin can be applied in development of electrochemical biosensors as a mimic peroxidase. However, because of the oxidative destruction and the self-aggregation of hemin to form catalytically inactive species, hemin in aqueous solution usually shows low catalytic activity [48]. Recently, graphene and G-quadruplex DNA oligomers have been considered as the successful carriers of hemin for the preparation of electrochemical sensors $[49,50]$. The hemin/graphene nanocomposites have also been utilized as the signal labels for electrochemical biosensing typically in a "sandwich-like" format. For example, Zhou et al. developed an electrochemical microRNAs biosensor with carboxylic graphene-hemin hybrid nanosheets as the labels, in which the nanosheets labeled with detection probe DNA for molecular recognition catalyzed the oxidation reaction of hydroquinone in the presence of $\mathrm{H}_{2} \mathrm{O}_{2}$ due to the intrinsic peroxidase-like activity of hemin on the carboxylic graphene surface [51]. $\mathrm{Li}$ et al. reported the amperometric detection of Escherichia 


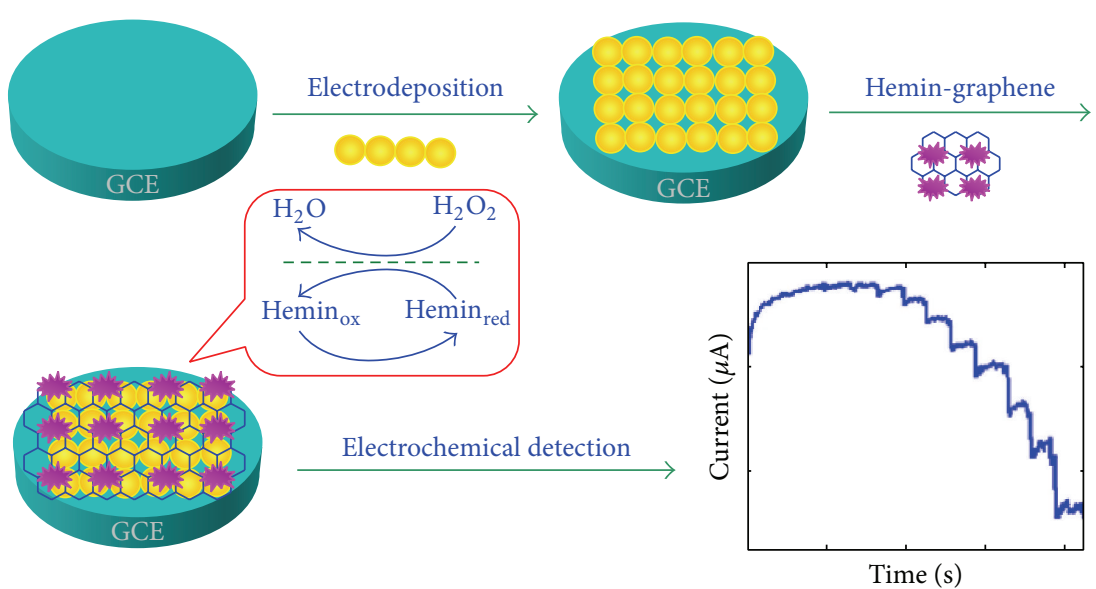

FIGURE 4: Preparation of the H-GNs/AuNP/GCE biosensor for the analysis of hydrogen peroxide followed by the electrochemical detection process and the output. (Reprinted with permission from [46]. Copyright 2013 Elsevier).
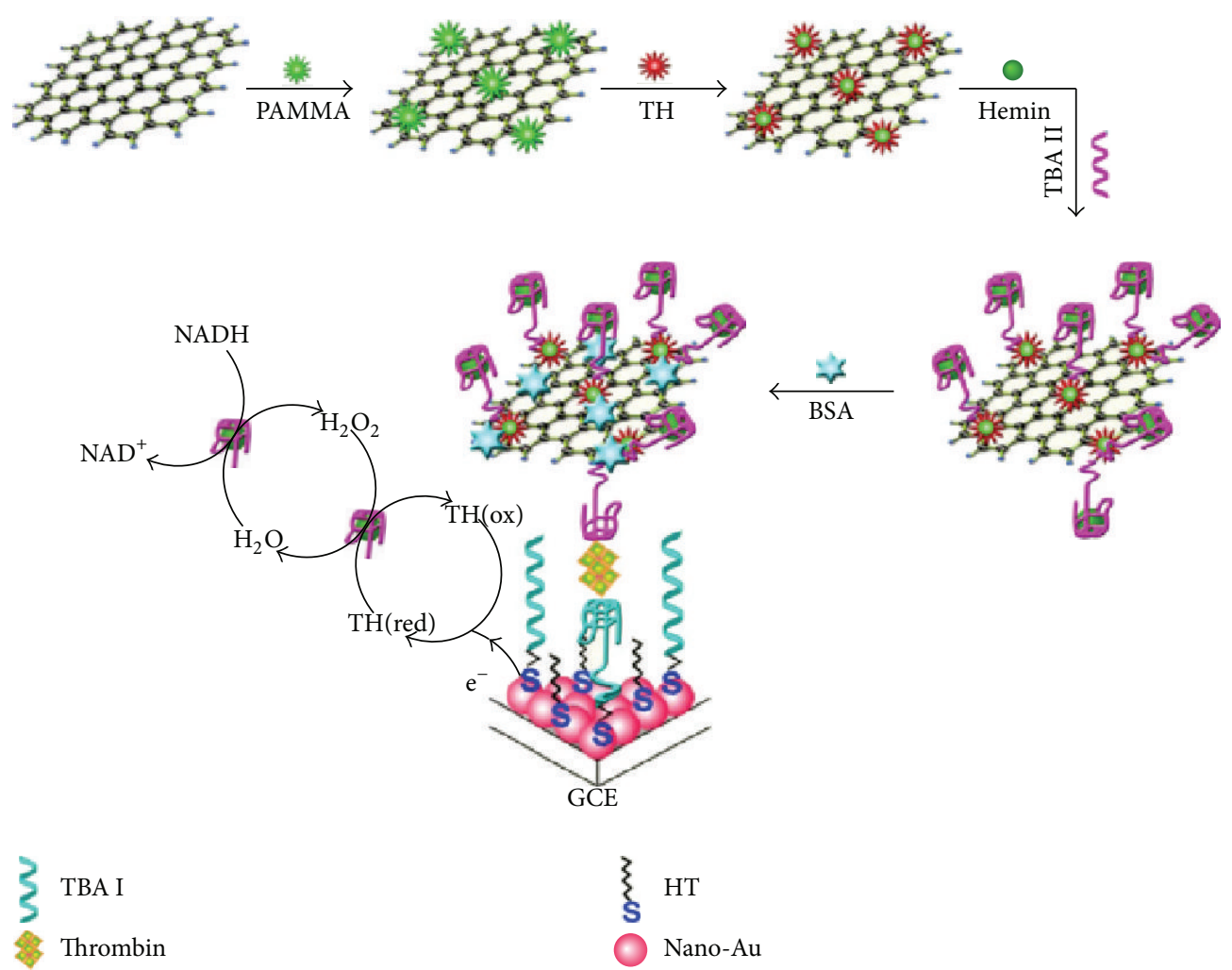

FIGURE 5: Schematic illustration of the pseudobienzyme aptasensor with PAMMA-rGO as nanocarrier and hemin/G-quadruplex as NADH oxidase and HRP-mimicking DNAzyme. (Reprinted with permission from [53]. Copyright 2013 Elsevier).

coli O157:H7 with the signal amplification of graphene-based nanosheets. The nanocomposites were prepared by electrostatic adsorption of thionine and AuNPs-coated $\mathrm{SiO}_{2}$ on GO, followed by assembly of signal DNA and G-quadruplex to form the hemin/G-quadruplex structure as HRP-mimicking DNAzyme [52]. Furthermore, as HRP-mimicking DNAzyme, the hemin/G-quadruplex structure can act as NADH oxidase simultaneously. For this consideration, Yuan's group developed three types of electrochemical biosensors for thrombin detection using the signal tags of dendrimer-rGO modified with thionine and hemin/G-quadruplex [53], AuNPs-rGO modified with alcohol dehydrogenase (ADH) and hemin/Gquadruplex [54], and rGO-Pd nanoparticles composites modified with $\mathrm{ADH}$ and hemin/G-quadruplex [55], respectively. As shown in Figure 5, the thionine- (TH-) conjugated hemin/G-quadruplex-polyamidoamine- (PAMAM-) rGO was captured on electrode surface. In the presence of $\mathrm{NADH}$, the hemin/G-quadruplex on electrode surface firstly acted as NADH oxidase, facilitating the oxidation of NADH to $\mathrm{NAD}^{+}$with the concomitant local formation of $\mathrm{H}_{2} \mathrm{O}_{2}$ in 
the presence of $\mathrm{O}_{2}$. Simultaneously, the hemin/G-quadruplex acted as an HRP-mimicking DNAzyme that quickly bioelectrocatalyzed the reduction of produced $\mathrm{H}_{2} \mathrm{O}_{2}$ with dramatic improvement of the oxidation-reduction reaction of the $\mathrm{TH}$ mediator, thus leading to an increase in the redox current of $\mathrm{TH}$.

\section{Conclusions and Outlook}

Recently, the application of ferrocene/graphene and hemin/graphene nanocomposites in electrochemical sensing is an important aspect of the graphene "gold rush." We, for the first time, reviewed the preparation and application of these nanocomposites in electrochemical sensing. For modification of ferrocene or its derivatives on graphene, $\pi-\pi$ stacking interaction, covalent bonding, and physical entrapment ionic liquid, polymers or sol-gel matrices are the commonly used strategies. Among them, covalent bonding is still the most popular method for the immobilization of ferrocene derivatives because it prevents effectively the leakage of electron shuttling ferrocene from electrode surface, thus enhancing the stability and sensitivity of the sensors. Overall, the ferrocene-functionalized graphene nanocomposites acting as the electrode materials have been used for the detection of clinical analytes (e.g., thrombin, cholesterol, uric acid, glucose, ascorbic acid, dopamine, hydrogen peroxide, and choline), drugs (e.g., acetaminophen, theophylline), and environmental pollutants like nitrite and dihydroxybenzene isomers. In the detection system, ferrocene could be regenerated by enzymes or different analytes after its electrooxidation. For grafting of hemin onto graphene, $\pi-\pi$ stacking interaction is the most popular method. Additionally, G-quadruplex DNA oligomers have been considered recently as the successful carriers for hemin to form mimic peroxidases. Hemin or hemin/Gquadruplex functionalized graphene nanocomposites act as HRP-mimicking DNAzyme in the detection system. Hemin/graphene and hemin/G-quadruplex/graphene nanocomposites have been used not only as the electrode materials to enhance the biocompatibility, electrocatalysis, and redox property of electrode but also as the signal tags for output. Moreover, the success of the initial efforts on preparation of graphene hybrid materials provides good motivation for further manipulation of ferrocene- and hemin-functionalized graphene/nanoparticles nanocomposites to improve sensing and catalytic efficiencies. We believe that this work would be valuable for the designing of graphene-based new types of electrochemical sensors.

\section{Conflict of Interests}

The authors declare that there is no conflict of interests regarding the publication of this paper.

\section{Acknowledgments}

Partial support of this work by the National Natural Science Foundation of China (nos. 21205003 and 21305004), the Joint
Fund for Fostering Talents of National Natural Science Foundation of China and Henan Province (U1304205), the Project of Education Department of Henan Province (14A150042), and the Program for Science and Technology Innovation Talents at the University of Henan Province (15HASTIT001) is gratefully acknowledged.

\section{References}

[1] D. A. C. Brownson, D. K. Kampouris, and C. E. Banks, "Graphene electrochemistry: fundamental concepts through to prominent applications," Chemical Society Reviews, vol. 41, no. 21, pp. 6944-6976, 2012.

[2] J. Liu, Y. Chen, Y. Guo, F. Yang, and F. Cheng, "Electrochemical sensor for o-nitrophenol based on $\beta$-cyclodextrin functionalized graphene nanosheets," Journal of Nanomaterials, vol. 2013, Article ID 632809, 6 pages, 2013.

[3] M. H. Chakrabarti, C. T. J. Low, N. P. Brandon et al., "Progress in the electrochemical modification of graphene-based materials and their applications," Electrochimica Acta, vol. 107, pp. 425440, 2013.

[4] Y. Liu, X. Dong, and P. Chen, "Biological and chemical sensors based on graphene materials," Chemical Society Reviews, vol. 41, no. 6, pp. 2283-2307, 2012.

[5] Y. Fang and E. Wang, "Electrochemical biosensors on platforms of graphene," Chemical Communications, vol. 49, no. 83, pp. 9526-9539, 2013.

[6] M. Pumera, "Graphene-based nanomaterials and their electrochemistry," Chemical Society Reviews, vol. 39, no. 11, pp. 41464157, 2010.

[7] H. Ma, D. Wu, Z. Cui et al., "Graphene-based optical and electrochemical biosensors: a review," Analytical Letters, vol. 46, no. 1, pp. 1-17, 2012.

[8] X. Huang, X. Qi, F. Boey, and H. Zhang, "Graphene-based composites," Chemical Society Reviews, vol. 41, no. 2, pp. 666686, 2012.

[9] V. Dhand, K. Y. Rhee, H. J. Kim, and D. H. Jung, "A comprehensive review of graphene nanocomposites: research status and trends," Journal of Nanomaterials, vol. 2013, Article ID 763953, 14 pages, 2013.

[10] F. Liu, C. W. Lee, and J. S. Im, "Graphene-based carbon materials for electrochemical energy storage," Journal of Nanomaterials, vol. 2013, Article ID 642915, 11 pages, 2013.

[11] S.-M. Chen, R. Ramachandran, V. Mani, and R. Saraswathi, "Recent advancements in electrode materials for the highperformance electrochemical supercapacitors: a review," International Journal of Electrochemical Science, vol. 9, no. 8, pp. 4072-4085, 2014.

[12] G. Kalita, S. Sharma, K. Wakita, M. Umeno, Y. Hayashi, and M. Tanemura, "A photoinduced charge transfer composite of graphene oxide and ferrocene," Physical Chemistry Chemical Physics, vol. 15, no. 4, pp. 1271-1274, 2013.

[13] M. B. Gholivand and M. Khodadadian, "Simultaneous voltammetric determination of captopril and hydrochlorothiazide on a graphene/ferrocene composite carbon paste electrode," Electroanalysis, vol. 25, no. 5, pp. 1263-1270, 2013.

[14] K. Deng, J. Zhou, and X. Li, "Noncovalent nanohybrid of ferrocene with chemically reduced graphene oxide and its application to dual biosensor for hydrogen peroxide and choline," Electrochimica Acta, vol. 95, pp. 18-23, 2013. 
[15] C. Jin, J. Lee, E. Lee, E. Hwang, and H. Lee, "Nonvolatile resistive memory of ferrocene covalently bonded to reduced graphene oxide," Chemical Communications, vol. 48, no. 35, pp. 42354237, 2012.

[16] R. S. Dey and C. R. Raj, "Redox-functionalized graphene oxide architecture for the development of amperometric biosensing platform," ACS Applied Materials \& Interfaces, vol. 5, no. 11, pp. 4791-4798, 2013.

[17] S. M. Liu, L. Wang, Y. Meng et al., “(4-Ferrocenylethyne) phenylamine functionalized graphene oxide modified electrode for sensitive nitrite sensing," Electrochimica Acta, vol. 116, pp. 504-511, 2014.

[18] B. Zhuo, Y. Li, X. Huang, Y. Lin, Y. Chen, and W. Gao, "An electrochemiluminescence aptasensing platform based on ferrocene-graphene nanosheets for simple and rapid detection of thrombin," Sensors and Actuators B: Chemical, vol. 208, pp. 518-524, 2015.

[19] L. Fan, Q. Zhang, K. Wang, F. Li, and L. Niu, "Ferrocene functionalized graphene: preparation, characterization and efficient electron transfer toward sensors of $\mathrm{H}_{2} \mathrm{O}_{2}$," Journal of Materials Chemistry, vol. 22, no. 13, pp. 6165-6170, 2012.

[20] M. B. Avinash, K. S. Subrahmanyam, Y. Sundarayya, and T. Govindaraju, "Covalent modification and exfoliation of graphene oxide using ferrocene," Nanoscale, vol. 2, no. 9, pp. 1762-1766, 2010.

[21] P. A. Azar, F. Farjami, M. S. Tehrani, and E. Eslami, "A carbon nanocomposite ionic liquid electrode based on montmorillonite nanoclay for sensitive voltammetric determination of thioridazine," International Journal of Electrochemical Science, vol. 9, no. 5, pp. 2535-2547, 2014.

[22] K.-J. Huang, Y.-X. Miao, L. Wang, T. Gan, M. Yu, and L.-L. Wang, "Direct electrochemistry of hemoglobin based on chitosan-ionic liquid-ferrocene/graphene composite film," Process Biochemistry, vol. 47, no. 7, pp. 1171-1177, 2012.

[23] S. Tajik, M. A. Taher, and H. Beitollahi, "Application of a new ferrocene-derivative modified-graphene paste electrode for simultaneous determination of isoproterenol, acetaminophen and theophylline," Sensors and Actuators, B: Chemical, vol. 197, pp. 228-236, 2014.

[24] S. A. Zaidi and J. H. Shin, "Molecularly imprinted polymer electrochemical sensors based on synergistic effect of composites synthesized from graphene and other nanosystems," International Journal of Electrochemical Science, vol. 9, no. 8, pp. 4598-4616, 2014.

[25] I. Cha, Y. Yagi, T. Kawahara et al., "Grafting of polymers onto graphene oxide by trapping of polymer radicals and ligandexchange reaction of polymers bearing ferrocene moieties," Colloids and Surfaces A: Physicochemical and Engineering Aspects, vol. 441, pp. 474-480, 2014.

[26] M. Liu, L. Wang, J. Deng et al., "Highly sensitive and selective dopamine biosensor based on a phenylethynyl ferrocene/graphene nanocomposite modified electrode," Analyst, vol. 137, no. 19, pp. 4577-4583, 2012.

[27] H. Peng, Z. Huang, Y. Zheng, W. Chen, A. Liu, and X. Lin, "A novel nanocomposite matrix based on graphene oxide and ferrocene-branched organically modified sol-gel/chitosan for biosensor application," Journal of Solid State Electrochemistry, vol. 18, no. 7, pp. 1941-1949, 2014.

[28] A. Fatoni, A. Numnuam, P. Kanatharana, W. Limbut, and P. Thavarungkul, "A novel molecularly imprinted chitosanacrylamide, graphene, ferrocene composite cryogel biosensor used to detect microalbumin," Analyst, vol. 139, no. 23, pp. 61606167, 2014.

[29] J. Han, Y. Zhuo, Y.-Q. Chai, L. Mao, Y.-L. Yuan, and R. Yuan, "Highly conducting gold nanoparticles-graphene nanohybrid films for ultrasensitive detection of carcinoembryonic antigen," Talanta, vol. 85, no. 1, pp. 130-135, 2011.

[30] M. Liu, J. Deng, Q. Chen et al., "Sensitive detection of rutin with novel ferrocene benzyne derivative modified electrodes," Biosensors and Bioelectronics, vol. 41, no. 1, pp. 275-281, 2013.

[31] M. Liu, Q. Chen, C. Lai et al., "A double signal amplification platform for ultrasensitive and simultaneous detection of ascorbic acid, dopamine, uric acid and acetaminophen based on a nanocomposite of ferrocene thiolate stabilized $\mathrm{Fe}_{3} \mathrm{O}_{4} @ \mathrm{Au}$ nanoparticles with graphene sheet," Biosensors and Bioelectronics, vol. 48, pp. 75-81, 2013.

[32] J. Liu, X. Wang, T. Wang et al., "Functionalization of monolithic and porous three-dimensional graphene by one-step chitosan electrodeposition for enzymatic biosensor," ACS Applied Materials \& Interfaces, vol. 6, no. 22, pp. 19997-20002, 2014.

[33] N. Huang, M. Liu, H. Li, Y. Zhang, and S. Yao, "Synergetic signal amplification based on electrochemical reduced graphene oxide-ferrocene derivative hybrid and gold nanoparticles as an ultra-sensitive detection platform for bisphenol A," Analytica Chimica Acta, vol. 853, pp. 249-257, 2015.

[34] P. Wan, S. Yin, L. Liu et al., "Graphene carrier for magnetocontrollable bioelectrocatalysis," Small, vol. 10, no. 4, pp. 647652, 2014.

[35] A. Wong and M. D. P. T. Sotomayor, "Determination of carbofuran and diuron in FIA system using electrochemical sensor modified with organometallic complexes and graphene oxide," Journal of Electroanalytical Chemistry, vol. 731, pp. 163171, 2014.

[36] Y. Ni, P. Wang, H. Song, X. Lin, and S. Kokot, "Electrochemical detection of benzo(a)pyrene and related DNA damage using DNA/hemin/nafion-graphene biosensor," Analytica Chimica Acta, vol. 821, pp. 34-40, 2014.

[37] Y. Guo, J. Li, and S. Dong, "Hemin functionalized graphene nanosheets-based dual biosensor platforms for hydrogen peroxide and glucose," Sensors and Actuators B: Chemical, vol. 160, no. 1, pp. 295-300, 2011.

[38] M. Zhang, R. Yuan, Y. Chai et al., "A cathodic electrogenerated chemiluminescence biosensor based on luminol and hemingraphene nanosheets for cholesterol detection," RSC Advances, vol. 2, no. 11, pp. 4639-4641, 2012.

[39] J. Zhang, W. Wang, S. Chen, Y. Ruo, X. Zhong, and X. Wu, "Bi-pseudoenzyme synergetic catalysis to generate a coreactant of peroxydisulfate for an ultrasensitive electrochemiluminescence-based cholesterol biosensor," Biosensors and Bioelectronics, vol. 57, pp. 71-76, 2014.

[40] A. A. Vernekar and G. Mugesh, "Hemin-functionalized reduced graphene oxide nanosheets reveal peroxynitrite reduction and isomerization activity," Chemistry, vol. 18, no. 47, pp. 1512215132, 2012.

[41] R. Jiang, D. T. Tran, J. McClure, and D. Chu, "Heat-treated hemin supported on graphene nanoplatelets for the oxygen reduction reaction," Electrochemistry Communications, vol. 19, no. 1, pp. 73-76, 2012.

[42] J. Wei, J. Qiu, L. Li et al., "A reduced graphene oxide based electrochemical biosensor for tyrosine detection," Nanotechnology, vol. 23, Article ID 335707, pp. 1-7, 2012. 
[43] R. Oprea, S. F. Peteu, P. Subramanian et al., "Peroxynitrite activity of hemin-functionalized reduced graphene oxide," Analyst, vol. 138, no. 15, pp. 4345-4352, 2013.

[44] Z. Tang, L. Gao, Y. Wu et al., "BSA-rGO nanocomposite hydrogel formed by UV polymerization and in situ reduction applied as biosensor electrode," Journal of Materials Chemistry $B$, vol. 1, no. 40, pp. 5393-5397, 2013.

[45] W. Lei, L. Wu, W. Huang, Q. Hao, Y. Zhang, and X. Xia, "Microwave-assisted synthesis of hemin-graphene/poly(3,4ethylenedioxythiophene) nanocomposite for a biomimetic hydrogen peroxide biosensor," Journal of Materials Chemistry B, vol. 2, no. 27, pp. 4324-4330, 2014.

[46] H. Song, Y. Ni, and S. Kokot, "A novel electrochemical biosensor based on the hemin-graphene nano-sheets and gold nanoparticles hybrid film for the analysis of hydrogen peroxide," Analytica Chimica Acta, vol. 788, pp. 24-31, 2013.

[47] Y. Zhang, Z. Xia, H. Liu, M. Yang, L. Lin, and Q. Li, "Hemingraphene oxide-pristine carbon nanotubes complexes with intrinsic peroxidase-like activity for the detection of $\mathrm{H}_{2} \mathrm{O}_{2}$ and simultaneous determination for Trp, AA, DA, and UA," Sensors and Actuators B: Chemical, vol. 188, pp. 496-501, 2013.

[48] T. Xue, S. Jiang, Y. Q. Qu et al., "Graphene-supported hemin as a highly active biomimetic oxidation catalyst," Angewandte Chemie International Edition, vol. 51, no. 16, pp. 3822-3825, 2012.

[49] Q. Wang, J. Lei, S. Deng, L. Zhang, and H. Ju, "Graphenesupported ferric porphyrin as a peroxidase mimic for electrochemical DNA biosensing," Chemical Communications, vol. 49, no. 9, pp. 916-918, 2013.

[50] I. Willner, B. Shlyahovsky, M. Zayats, and B. Willner, "DNAzymes for sensing, nanobiotechnology and logic gate applications," Chemical Society Reviews, vol. 37, no. 6, pp. 1153-1165, 2008.

[51] Y. Zhou, M. Wang, Z. Xu, C. Ni, H. Yin, and S. Ai, "Investigation of the effect of phytohormone on the expression of microRNA159a in Arabidopsis thaliana seedlings based on mimic enzyme catalysis systematic electrochemical biosensor," Biosensors and Bioelectronics, vol. 54, pp. 244-250, 2014.

[52] Y. Li, J. Deng, L. Fang et al., "A novel electrochemical DNA biosensor based on HRP-mimicking hemin/G-quadruplex wrapped GOx nanocomposites as tag for detection of Escherichia coli O157:H7," Biosensors and Bioelectronics, vol. 63, pp. 1-6, 2015.

[53] Y. Yuan, G. Liu, R. Yuan, Y. Chai, X. Gan, and L. Bai, "Dendrimer functionalized reduced graphene oxide as nanocarrier for sensitive pseudobienzyme electrochemical aptasensor," Biosensors and Bioelectronics, vol. 42, no. 1, pp. 474-480, 2013.

[54] H. Yi, W. Xu, Y. Yuan et al., "A pseudo triple-enzyme cascade amplified aptasensor for thrombin detection based on hemin/G-quadruplex as signal label," Biosensors and Bioelectronics, vol. 54, pp. 415-420, 2014.

[55] S. Xie, Y. Chai, R. Yuan, L. Bai, Y. Yuan, and Y. Wang, "A dual-amplification aptasensor for highly sensitive detection of thrombin based on the functionalized graphene-Pd nanoparticles composites and the hemin/G-quadruplex," Analytica Chimica Acta, vol. 755, pp. 46-53, 2012. 

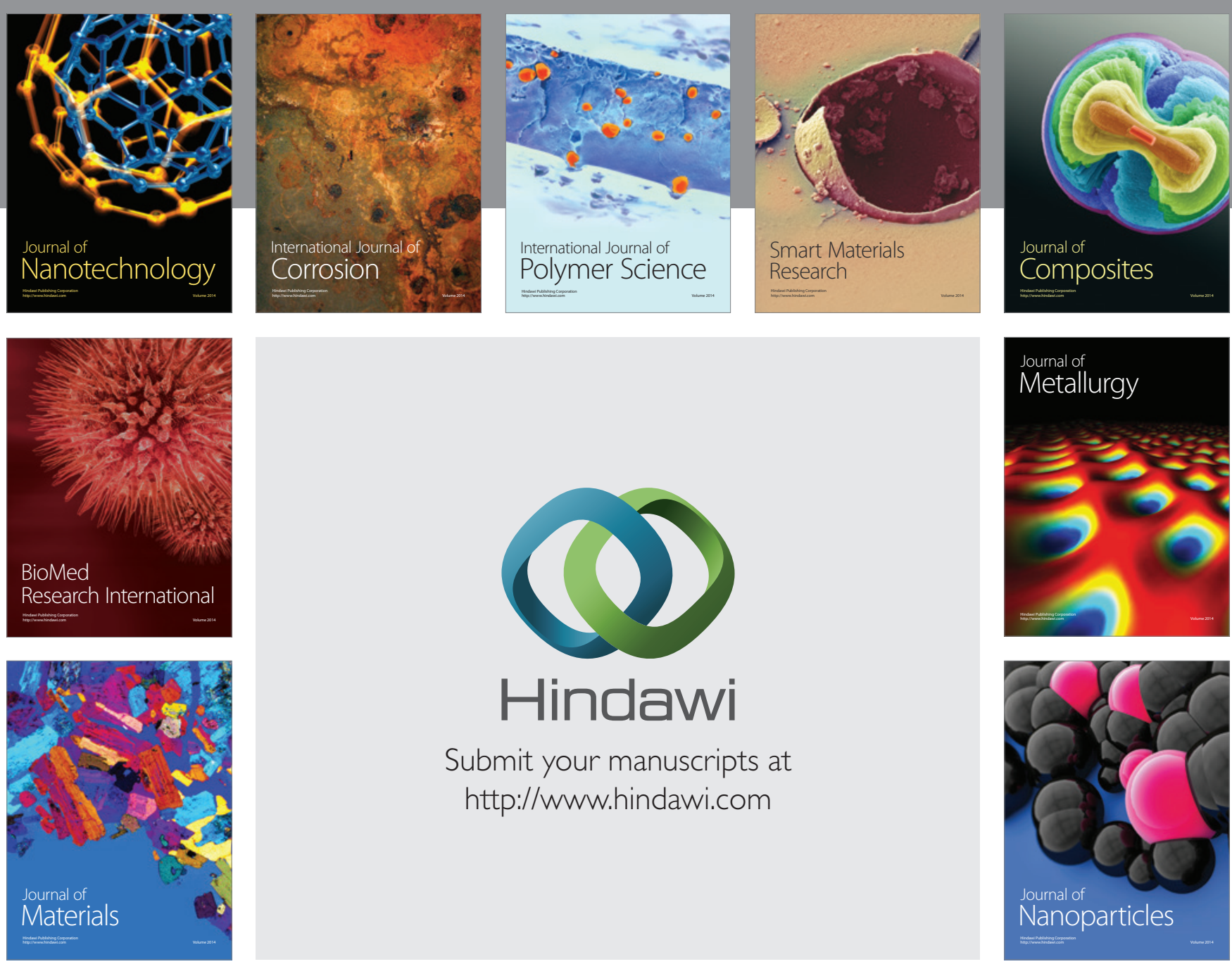

Submit your manuscripts at http://www.hindawi.com
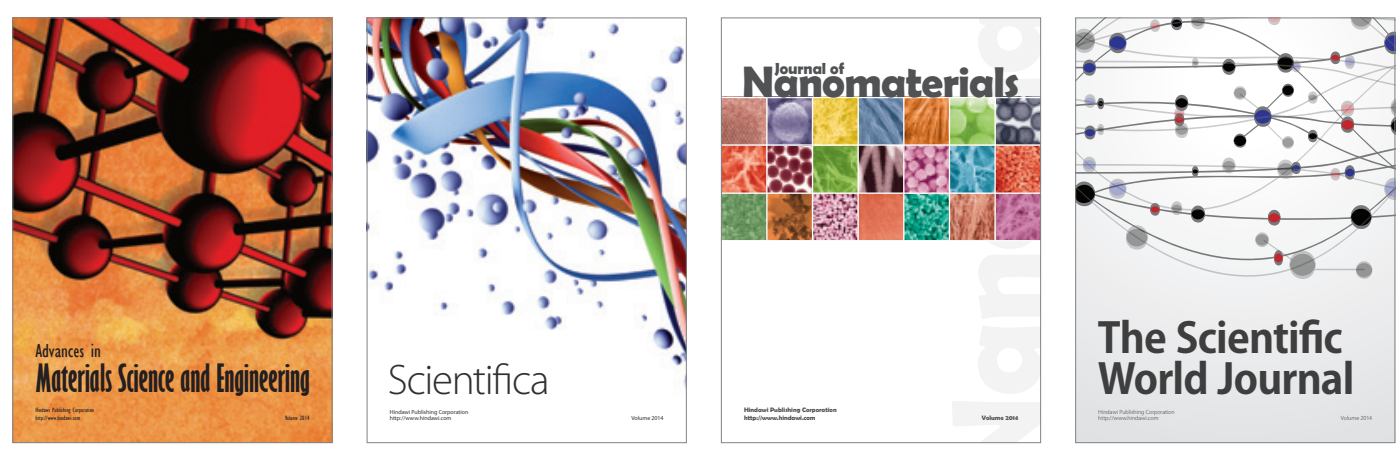

\section{The Scientific World Journal}
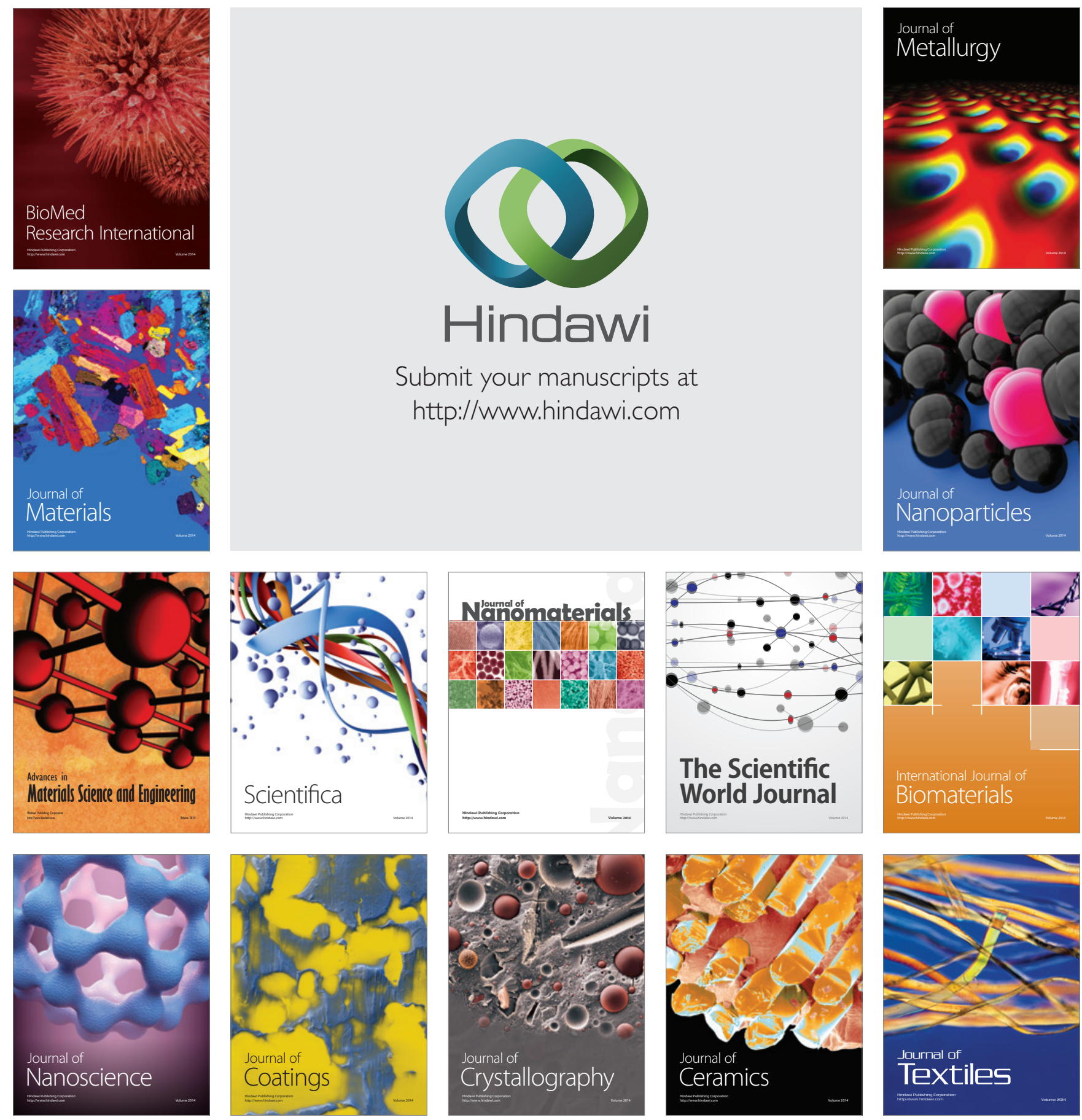